\title{
Stimmfreigabe zur öffentlichen Krankenkasse
}

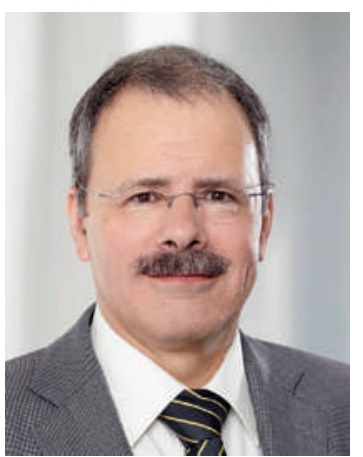

Frühestens am 28. September 2014 und spätestens am 8. März 2015 wird voraussichtlich die Volksabstimmung über die Initiative «Für eine öffentliche Krankenkasse» stattfinden.

Die unterschiedlichen Positionen zu diesem Thema, über die mit näherrückendem Abstimmungstermin auch in der Öffentlichkeit noch lebhafte Diskussionen zu erwarten sind, zeichneten sich in der FMH spätestens seit Anfang diesen Jahres ab.

Die Vernehmlassung, die die FMH im Februar und März 2014 unter den ihr angeschlossenen Ärzteorganisationen durchführte, zeigte ein sehr gemischtes Bild. So schickten einige Ärzteorganisationen Argumentarien, die eine NeinPosition ausführlich begründeten, und gaben ihrer Sorge über möglicherweise fatale Auswirkungen einer monopolistischen «Einheitskasse» Ausdruck. Andere Verbände erläuterten ebenso ausführlich ihre Pro-Argumente und betonten, dass mit einer «öffentlichen Krankenkasse» das Problem der Risikoselektion endlich vollständig behoben werden könne und das Gesundheitswesen solidarischer gestaltet würde.

Die Mehrzahl der Rückmeldungen beinhaltete jedoch weder ein klares «Pro» noch ein eindeutiges «Contra» zur Initiative, sondern verwies auf die sehr geteilten Meinungen innerhalb des jeweiligen Fach- oder Kantonsverbands. Einige dieser Ärzteorganisationen machten gegenüber der FMH auch explizit ihre Auffassung deutlich, dass man nur mit

\section{Nur eine Stimmfreigabe kann den divergierenden Meinungen zwischen aber auch innerhalb der einzelnen Verbände gerecht werden.}

einer Entscheidung der Ärztekammer für eine Stimmfreigabe den bestehenden Differenzen Rechnung tragen und einzelnen Verbänden die andernfalls zu erwartenden Zerreissproben ersparen könne.

Angesichts der sehr divergierenden Meinungen zwischen den verschiedenen Organisationen, aber auch innerhalb der einzelnen Verbände empfahlen sowohl der Zentralvorstand als auch die Delegiertenversammlung der Ärztekammer eine Stimmfreigabe zur Volksinitiative «Für eine öffentliche Krankenkasse».
Nicht zuletzt weil die Volksinitiative eine für das Schweizer Gesundheitswesen wegweisende Entscheidung betrifft, sollte eine Positionierung der FMH für oder gegen «eine öffentliche Krankenkasse» eine breite Unterstützung der Basis geniessen. Gäbe die FMH eine Stellungnahme zu einer solch entscheidenden Weichenstellung des Gesundheitssystems lediglich aufgrund einer knappen Mehrheit ab, würde sie nicht nur ihren eigenen inneren Zusammenhalt und denjenigen einzelner Ärzteverbände schwächen. Sie würde auch ihre Glaubwürdigkeit nach aussen verspielen, wenn ihre offizielle Position von vielen Ärztinnen und Ärzten nicht mitgetragen oder sogar eindeutig abgelehnt würde.

Die Diskussion in der Ärztekammer vom 8. Mai mit vielen Beiträgen von Befürwortern wie Gegnern der Initiative spiegelte die oben beschriebene Situation gut wider. Dem

\section{Eine Positionierung der FMH zu einer solch wegweisenden Entschei- dung für das Schweizer Gesundheits- wesen sollte eine breite Unterstützung der Basis geniessen.}

Wunsch, zunächst eine umfassende Diskussion zur Sache zu führen, wurde unter anderem entgegengehalten, dass Auseinandersetzungen zu diesem Thema leider oftmals wenig sachlich verliefen. Diese ähnelten teilweise einer Konfrontation der «Religionen», in denen man einander die Extreme zentralistischer Regelungswut bzw. profitorientierten Liberalismus vorwerfe. Neben diesem Dissens zeichnete sich jedoch auch ein breiter Konsens der Delegierten ab, nämlich dass auf Seiten von Befürwortern wie Gegnern der Initiative eine grosse Unzufriedenheit mit dem aktuellen System besteht und Verbesserungen gewünscht werden. Besonders pointiert wurde dies in einem Wortbeitrag deutlich, der die Wahl «Pro» und «Contra» öffentliche Krankenkasse als eine Wahl zwischen Pest und Cholera bezeichnete.

Die Ärztekammer hat nach einer engagierten - aber nicht ideologischen - Diskussion mit 85\% der Stimmen den Antrag auf Stimmfreigabe zur Initiative «Für eine öffentliche Krankenkasse» befürwortet. Die FMH nimmt somit keinen Einfluss auf die Stimmbürger, wenn es zwischen den Argumenten der «Pro»- und der «Contra»-Seite abzuwägen gilt und lässt den angeschlossenen Verbänden, die eine klare Positionierung wünschen, die Möglichkeit, für oder gegen eine «öffentliche Krankenkasse» einzutreten.

Dr. med. Jürg Schlup, Präsident der FMH 